\title{
EL GENERO BRIMEURA SALISB. EN LAS ISLAS BALEARES
}

\author{
G. BIBILONI, L. LLORENS \& J. RITA
}

\begin{abstract}
RESUMEN: Se comenta la situación en Mallorca de Brimeura fastigiata (Viv.) Salisb. y de Brimeura amethystina (L.) Chouard. Esta isla balear es el único territorio donde se conoce la coexistencia de estas dos especies consideradas, tradicionalmente, como vicariantes.

Se detalla el proceso de formación de bulbilos foliares en Br. fastigiata. Dichos bulbilos se desarrollan con posterioridad al desprendimiento de los ápices de las hojas.

Asimismo, se comentan las analogías que se han observado entre el desarrollo de una plántula a partir de un bulbilo y de la germinación de una semilla de liliáceas de tipo hipogeo.
\end{abstract}

RESUME: On discute la situation de Brimeura fastigiata (Viv.) Salisb. et Brimeura amethystina (L.) Chouard à Majorque. Cette île balear c'est l'unique endroit connue où vivent les deux espèces ansemble, considérés jusqu'à maintenant comme vicariantes.

On detaille le procès de formation des bulbilles foliaires chez Br. fastigiata. Ces bulbilles on développement après la chute spontanée des bouts des feuilles.

On commente, en fin, les analogies observés entre le développement d'une plantule naissant d'une bulbille et la germination d'une semence de Liliacée hypogée.

\section{INTRODUCCION}

Las especies del género Brimeura Salisb. han tenido un tratamiento nomenclatural complejo ya que han sido adscritas, en repetidas ocasiones, a otros géneros, especialmente a Hyacinthus y Scilla. Gracias a los trabajos de Chouard (1931, 1933) Y Garbari (1966, 1970) está ampliamente aceptado que Brimeura es un género independiente dentro de Liliaceae (Molero, 1975; Vosa, 1979; Heywwod, 1980; Pignati, 1982, (III):374). Además de diversos caracteres morfológicos, el número cromosómico $(n=14)$ separa claramente a Brimeura de $10 s$ otros géneros próximos (Garbari, 1970:25).

Brimeura es un género que tradicionalmente se ha considerado biespecífico, con $\mathrm{Br}$. amethystina (L.) Chouard (=Hyacinthus amethystinus L.) y Br. fastigiata (Viv.) Salisb. (=Scilla fastigiata Viv., = Hyacinthus fastigiatus (Viv.) Bert., झ Hyacinthus pouzolzii Gay). Parece probable que ambas especies se diferenciaron a partir de una única especie que, a su vez, pudo tener un origen hibrido (Garbari, 1970:33). Algunas poblaciones tetraploides han sido consideradas a nivel 
especifico: Br. fontqueri (Pau) Speta (Speta, 1982), aunque previamente se habia criticado la validez de esta especie dada la inconsistencia de los caracteres morfológicos en que se basaba la descripción de Pau (Molero, 1975:358).

\section{OBSERVACIONES BIOGEOGRAFICAS Y ECOLOGICAS}

Brimeura amethystina tiene un área de distribución disyunta. Considerada como un endemismo pirenaico, se encontró más tarde en los Ports de Tortosa y en el Montsan, sierras que forman el extremo meridional de la Cordillera Prelitoral Catalana (Cadeva11, 1913-1937; Molero, 1975) y también en el Monte Kapela y Jelenica, en el norte de Yugoslavia (Garbari, 1970) (fig. 1). Recientemente ha sido encontrada en una estación de Mallorca (islas Baleares) (figs. 1 y 2 ). Está última población se ha descrito como var. duvigneaudii Llorens (Llorens, 1984).

Brimeura fastigiata es una especie que ha sido considerada como "tirrénica" ya que sólo se la conocía en algunas islas del Mediterráneo Occidental (Córcega, Cerdeña, Menorca y otras menores). Pero recientemente ha sido hallada también en los Tagetes (Peloponeso, Grecia) (Damboldt et al., 1976).

La presencia de esta especie en Menorca se conoce desde hace años en diversas localidades de la zona norte de la isla (S'Enclusa, Fornel1s, Algaiarens, Binime1.1à) (Porta, 1887; Rodriguez, 1904; Montserrat, 1953). En material recolectado en esta isla se han contado $2 \mathrm{n}=42$ cromosomas por 10 que se la considera una población, al menos en parte, triploide (Vossa, 1979). Recientemente 1 a hemos encontrado en Mallorca, en una localidad situada en el SE de la isla próxima a Cala Pi (figs. 1 y 2). Una cita anterior de esta especie en Mallorca corresponde, de hecho, a una confusión con Br. amethystina var. duvigneaudii (Llorens, 1984:55). Ambas especies que hasta ahora han sido consideradas como alopráticas coinciden en Mallorca, aunque sea en áreas geográficas diferentes.

Brimeura amethystina es una especie que tiene un carácter montano o subalpino, desarrollándose especialmente en prados y en altitudes situadas por encima de los $1200 \mathrm{~m}$. En Mallorca se comporta de un modo diferente, ya que crece en las grietas de un acantilado maritimo, orientado al NW y a sólo $120 \mathrm{~m}$ sobre el nivel del mar. En esta localidad se ha observado que la reproducción de las plantas es preferentemente asexual mediante bulbilos. No se ha apreciado, durante los tres años en que han sido observada's (tanto en el campo como en cultivo), ni la formación de bulbilos foliares, ni tampoco la formación de cápsulas y semillas.

Brimeura fastigiata parece desarrollarse en condiciones más heterogéneas, no son raras la citas de localidades próximas al mar (Martinoli, 1949:679; Garbari, 1970:27). En Mallorca se halla a una altura de unos $10 \mathrm{~m}$ sobre el nivel del mar en un territorio que posee un clima mediterráneo semiárido, con unas precipitaciones anuales inferiores a $400 \mathrm{~mm}$. El sustrato geológico está formado por dunas cuaternarias consolidadas, establecidas sobre rocas miocénicas, que han dado lugar a un suelo carbonatado, con una textura areno-limosa. La única población conocida se encuentra en una estrecha franja de unos $40 \mathrm{~m}$, en el talud que bordea un pequeño barranco. La mayor parte de los individuos están situados sobre una pendiente orientada al norte y en una posición que las resguarda de la 


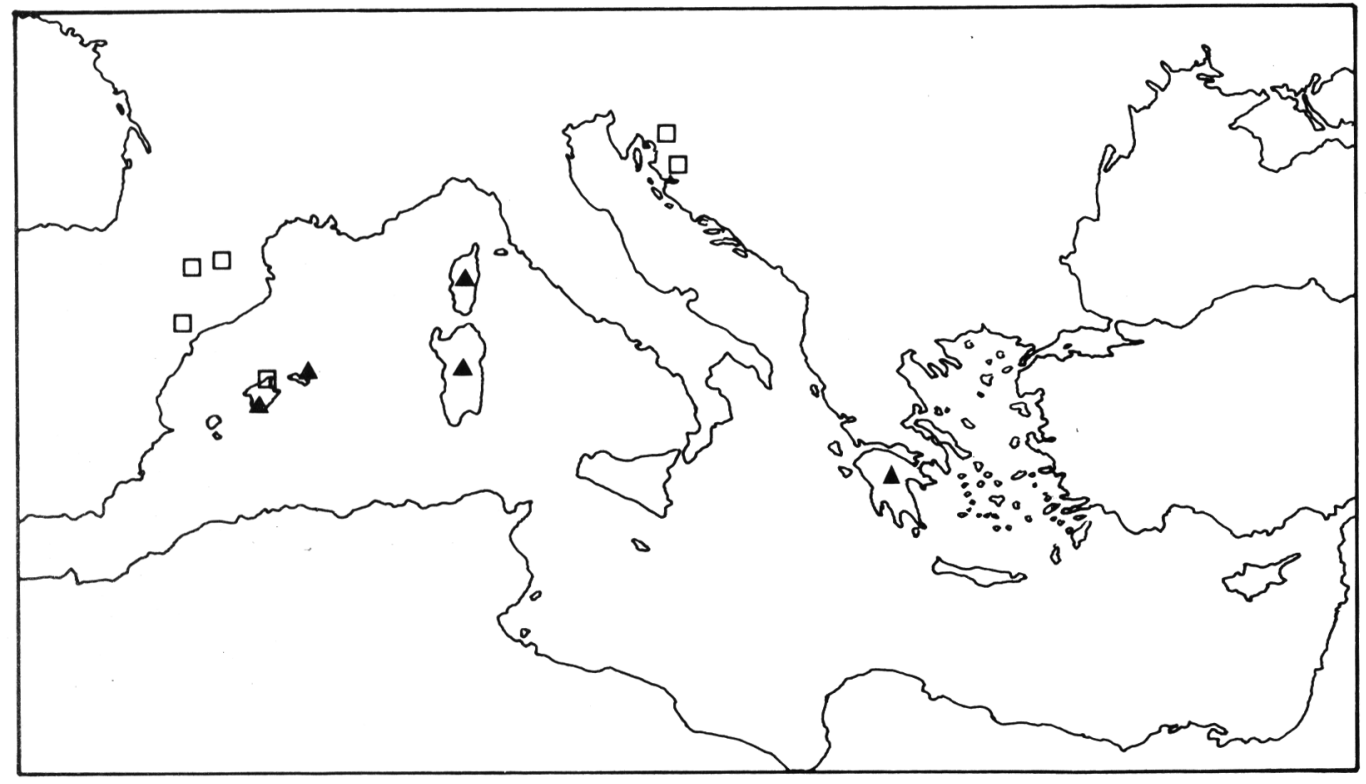

Figura 1.- Distribución conocida de Brimeura fastigiata (Viv.) Salisb. ( $($ ) y de Br. amethystina (L.) Chouard ( $\square$ ).

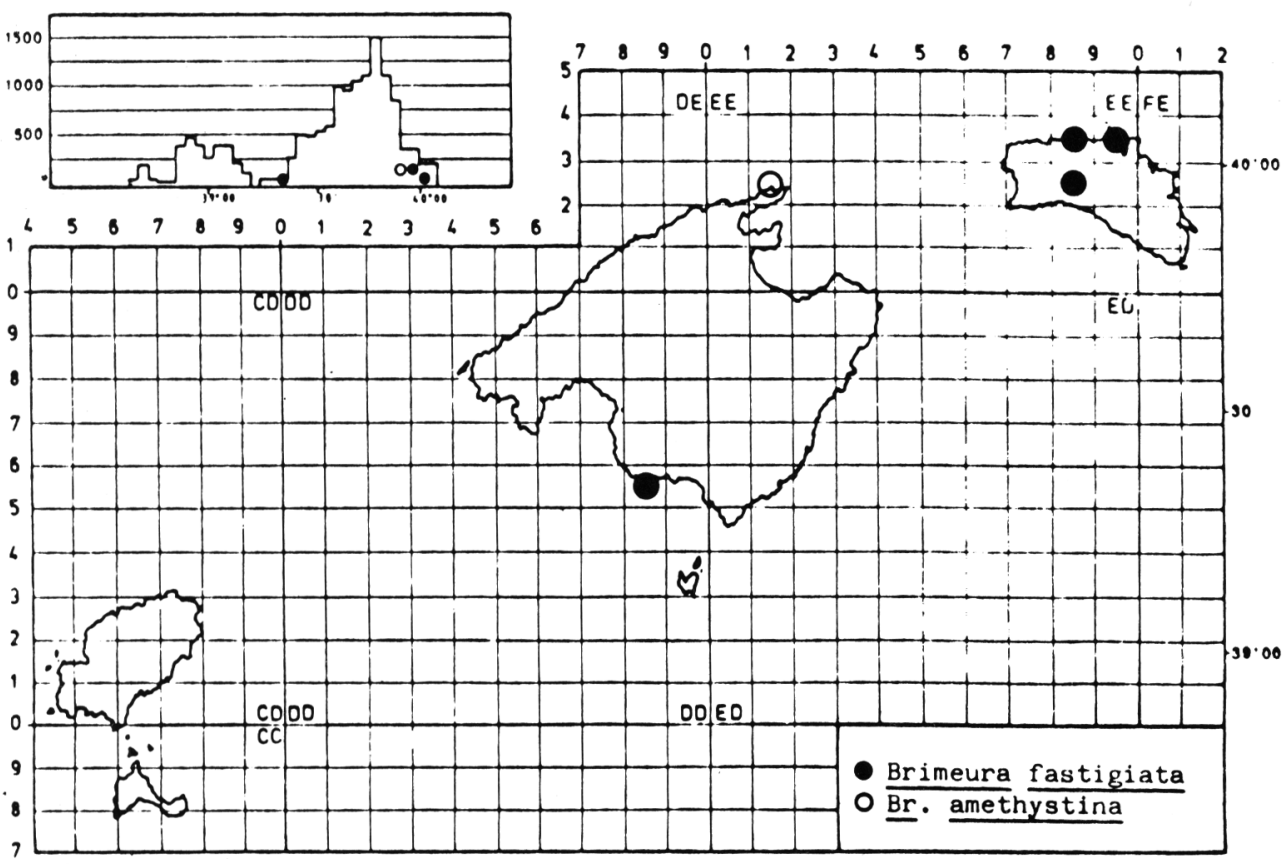

Figura 2.- Distribución conocida en las Islas Baleares de Brimeura fastigiata (Viv.) Salisb. $y$ de Br. amethystina (L.) Chouard. 
luz solar directa. El suelo que ocupan está notablemente humedecido desde las primeras lluvias otoñales hasta la primavera, ya que por esta zona drena el agua que se infiltra en las cotas superiores. En Mallorca se comporta como una especie esciófila y mesófila, pese a que se encuentra bajo un clima general mediterráneo semiárido. Esta misma ecología ha sido descrita para las estaciones próximas al mar en Cerdeña (Martinoli, 1949:679) y en Córcega (Garbari, 1970:27).

La vegetación que acompaña a $\mathrm{Br}$. fastigiata en Mallorca es un matorral mixto de las alianzas oleo-Ceratonion y Rosmarino-Ericion con un arbolado de Pinus halepensis. E1 estrato herbáceo pertenece a la clase Thero-Brachypodietea.

\section{OBSERVACIONES SOBRE EL CICLO BIOLOGICO DE BRIMEURA FASTIGIATA}

La reproducción de $\mathrm{Br}$. fastigiata en Mallorca, es casi exclusivamente agámica; aunque algunos individuos llegan a florecer, no se ha observado la formación de fructificaciones. Sin embargo, en dos años consecutivos se ha constatado el desprendimiento, de forma espontánea, de numerosos ápices foliares que, una vez en el suelo, han desarrollado bulbilos. Este fenómeno ha sido observado en su medio natural en las poblaciones de Menorca y en individuos cultivados procedentes de Córcega y Cerdeña (Garbari, com. pers.). Dado el interés de este peculiar tipo de reproducción la describimos con más detalle a continuación.

La reproducción asexual de esta especie se conoce desde el siglo pasado, e incluso fué descrita la var. bulbillifera Par1., en base a un único carácter diferencial que era la producción de bulbilos foliares. Esta variedad fué desestimada más tarde (Martinoli, 1949; Garbari, 1970), al comprobarse que la formación o no de dichos bulbilos dependia de las caracteristicas del medio donde residian las plantas y que, por tanto, el carácter no tenía consistencia para diferenciar un taxon. Más tarde Chouard (1931), al revisar el género Brimeura, consideró la capacidad para formar bulbilos foliares como un carácter propio del género.

Pese a que este tipo de reproducción es citado repetidamente en la bibliografia, no se ha encontrado una descripción detallada del mismo y algunos de los datos que han sido publicados no coinciden con nuestras observaciones. Chouard (1931:250) indica que en Brimeura fastigiata: "1a régénération se produit sur les feuilles au niveau du moindre traumatisme et sans qu'elles soient détachés du bulbe". Por su parte Martinoli (1949:684), también da a entender que 1 a producción de bulbilos tiene lugar sobre las hojas. Garbari (1970) en su estudio monográfico sobre Brimeura, consiguió la formación de bulbilos tanto sobre hojas enteras separadas del bulbo, como en secciones distales de las hojas y en trozos de hojas sin ápices, mantenidas a temperaturas y humedad controladas en el laboratorio, pero no describe el proceso de formación de los bulbilos en la naturaleza. Sin embargo, este autor ha observado el desprendimiento natural de los ápices y la fórmación de bulbilos en ellos (Garbari, 1966:325), pero hace extensivo este proceso a $\mathrm{Br}$. amethystina (hecho que nosotros no hemos observado en Mallorca).

Este autor nos ha confirmado (in litt.) que el mecanismo de formación de los bulbilos que ha podido observar coincide básicamente con el que describimos en este articulo. 

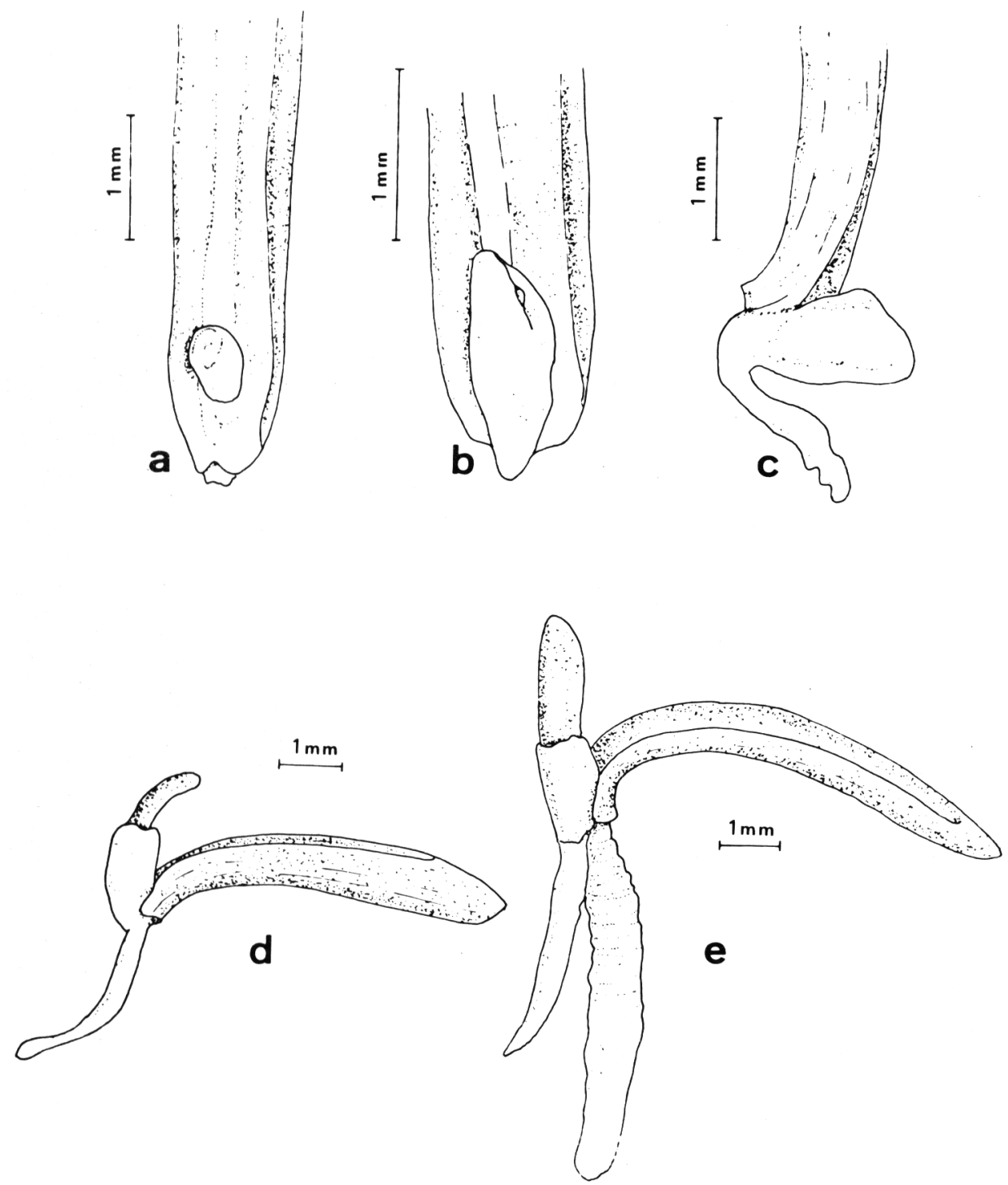

Figura 3.- Fases de desarrollo de los bulbilos que aparecen sobre los ápices desprendidos de las hojas de Brimeura fastigiata (Viv.) Salisb. a) Diferenciación de un meristema en la base ael ápice. b) Desarrollo del bulbilo. c) Formación de la primera raíz. d) Inicio del crecimiento de la primera hoja. e) Desarrollo de la segunda raiz. Todas las escalas corresponden a $1 \mathrm{~mm}$. 
Se ha seguido el comportamiento de Br. fastigiata, en la única localidad conocida hasta la fecha en Mallorca, a partir de la primavera de 1984, cuando fué hallada, hasta el verano de 1985. El seguimiento se ha llevado a cabo tanto en el campo, como en el laboratorio, con el cultivo en macetas de plantas enteras y de ápices y bulbilos en placas Petri. Estas últimas se mantuvieron a temperatura ambiente y sobre un sustrato de arena de mar lavada, permanentemente humedecido, salvo en la última fase, con una solución nutritiva.

E1 ciclo anual se incia a principios de octubre con el desarrollo de las primeras hojas (proceso que puede extenderse a lo largo de un mes). Antes de que las hojas se hayan desarrollado totalmente, y en un plazo que puede ser inferior a diez días, aparecen constricciones a una distancia de $6-8 \mathrm{~mm}$ de los ápices de todas las hojas de la mayor parte de las plantas. Tanto el desarrollo de las hojas como la aparición de septos se dan cincrónicamente en su medio natural y en las macetas. Se ha observado, sin embargo, que los individuos que se desarrollan tardiamente no presentan dichas constricciones. Una vez ise han formado los septos, una pequeña sacudida de la hoja provoca el desprendimiento de los ápices.

Al cabo de 10 a 20 dias, en la cara’ventral de 10 śpices desprendidos, ligeramente por encima de la zona de fractura, empieza a destacarse un meristema formado por células redondeadas, blancas y traslúcidas. Las células de este meristema se multiplican rápidamente y dan lugar a un bulbilo, más o menos ovalado, hueco y abierto por su parte superior. Este bulbilo ocupa toda la base del ápice y mide hasta $2,5 \mathrm{~mm}$ ( fig. 3).

Durante este periodo en se que forma el bulbilo el ápice se mantiene turgente, por 10 que debe suponerse que es capaz de absorber agua a través de la cuticula. •

Antes de que el bulbilo alcance su máximo desarrollo se diferencia en la base del mismo una protuberancia que rápidamente da lugar a una pequeña raíz. Al final del mes de noviembre se pudo observar cómo la mayor parte de los ápices desprendidos habian 11 egado a esta fase.

Si la raiz logra penetrar en el suelo y encuentra un medio húmedo y favorable, en la parte interna del bulbilo se diferencia un tejido clorofilico. Este tejido se desarrolla rápidamente y da lugar a una hoja (esporádicamente pueden aparecer dos), se sección circular, que sobresale por la abertura apical del bulbilo. Si las condiciones favorables se mantienen el crecimiento de la hoja es muy rápido, en pocos días puede alcanzar uno o dos centimetros.

Al mismo tiempo que crece la hojita, de la base del bulbilo se diferencia una pequeña raiz, en este caso adventicia. Esta raiz es maś vigorosa que la primera y presenta en su base engrosamientos anulares, por 10 que probablemente es de tipo contrácti1 o "tectriz" (Chouard, 1933:189), análogas a las que aparecen en las plántulas originadas a partir de semillas.

En algunos de los ápices cultivados en el laboratorio se ha observado la aparición de dos o más raíces adventicias. Estos casos, al igual que las malformaciones en los bulbilos, son más frecuentes sobre las secciones de hojas o sobre las hojas enteras que en los ápices desprendidos de forma natural.

El proceso finaliza, pués, con la formación de un pequeño bulbo que ha penetrado en el suelo. Al llegar el período seco esta plántula pierde la hoja y se mantiene inactiva hasta el otoño. 


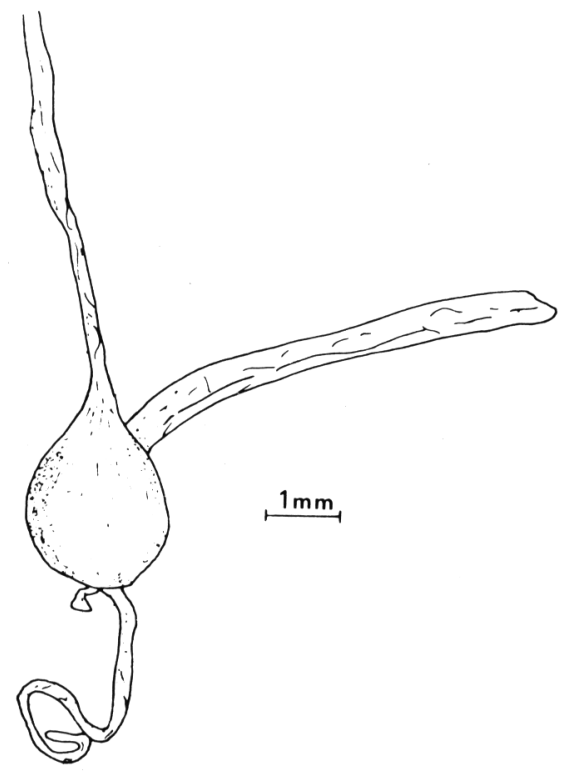

Figura 4.- Aspecto del bulbilo una vez que se ha desecado la plántula. Estas estructuras pueden perdurar en la superficie del suelo a lo largo del verano.
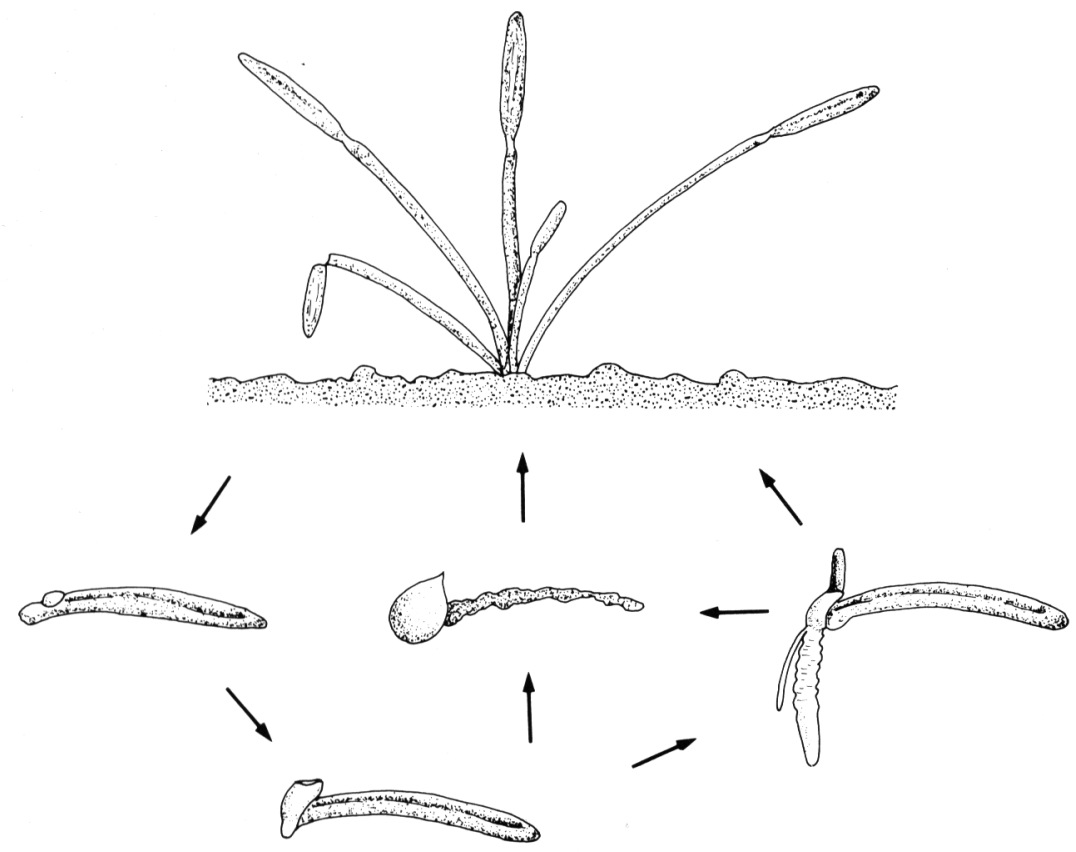

Figura 5.- Esquema del ciclo de reproducción asexual de Brimeura fastigiata (Viv.) Salisb. mediante bulbilos foliares. 
En los casos en que la primera raíz no logra penetrar el suelo, o no profundiza lo suficiente para evitar la superficie desecada del mismo, queda abortado el desarrollo de la hoja y raíz. En estos casos se produce un engrosamiento del bulbilo, se cierra su parte apical, mientras que la raicilla y el ápice que los soporta se marchitan. Bulbilos de este tipo recogidos en la primavera de 1984, y mantenidos fuera de su ambiente habitual durante el verano fueron capaces de desarrollar una nueva raiz y hoja al depositarlos sobre un sustrato humedecido con solución nutritiva. Así pués, estos bulbilos tienen capacidad para resistir el largo período desfavorable en la superficie del suelo y reactivarse con la humedad que producen las lluvias otoñales.

También se ha observado, en ápices que se desarrollaron en cajas de Petri con solución nutritiva, que una vez formadas la hoja y la raiz adventicia, si se deja desecar el medio de cultivo, el bulbilo es capaz de engrosarse mientras se marchita la hoja recién formada. Por lo tanto, el hecho de desarrollar la hoja no impide que el bulbilo revierta en un órgano de resistencia si las condiciones ambientales se vuelven desfavorables (figs. 4 y 5 ).

\section{CONCLUSIONES}

Mallorca es el único territorio donde se conoce la presencia de las dos especies del género Brimeura. Este hecho tiene interés biogeográfico ya que hasta ahora se había considerado a Br. amethystina y Br. fastigiata como especies vicariantes originadas a partir de un ancestro común. Sin embargo, la situación simpátrica de estas especies en Mallórca debe ser matizada, ya que, hasta el momento, sóló conocemos una localidad para cada una de ellas y en ambos casos de una extensión muy reducida. Estas estaciones, además, se hallan a considerable distancia entre ellas y en ambientes diferentes.

La multiplicación vegetativa mediante bulbilos es un fenómeno muy común en Liliaceae, también se sabe que muchas especies de esta familia son capaces de desarrollar tejidos embrionarios a partir de tejidos diferenciados, pero por 10 regular este proceso tiene lugar cuando ha ocurrido algún traumatismo sobre la planta (Camefort, 1972). A este fenómeno Chouard (1933) 1o denominó "regeneration" para diferenciarlo de "bourgeonnement" que es un proceso activo de reproducción asexual por división del bulbo. La regeneración a partir de hojas verdes es rara, pero puede observarse entre otras especies en Scilla verna Hudson, Hyacinthoides non-scripta (L.) Hoffmanns \& Link y H. hispanica (Miller) Rother., y en las dos especies de Brimeura (Chouard, 1933).

El proceso de reproducción vegetativa que hemos observado en Brimeura fastigiata se aparta significativamente del modelo general, ya que la producción de bulbilos no dependè de la actuación de un agente externo que provoque un traumatismo, sino que la rotura y desprendimiento de los ápices es controlado por mecanismos internos de las plantas, se puede dar o no según las condiciones ambientales donde viven. Por otra parte, los bulbilos formados son capaces de resistir una estación desfavorable, y reactivarse al 1 legar el otoño, por 10 que no sólo deben considerarse como un sistema de "regeneración" sino también como órganos de resistencia y de dispersión. 
Es destacable la analogía que hay entre el desarrollo de una nueva planta a partir de un bulbilo apical, y a partir de la germinación de semillas de Liliáceas de tipo hipogeo. En este caso la hoja cotiledónica es análoga morfológica y funcionalmente al'bulbilo foliar.

Por otra parte, la formación de plántulas o bulbilos en los ápices foliares desprendidos también tiene marcadas similitudes con el cultivo de tejidos vegetales "in vitro" a partir de tejidos diferenciados. En ambos casos se produce una desdiferenciación celular que permite la formación de masas embrionarias a partir de células diferenciadas (Camefort, 1972; Gaussen el al., 1982).

En el caso que nos ocupa parece probable que el ápice desprendido actúe a modo de planta "nurse", 1 a cual provee de nutrientes y regula la producción de hormonas necesaria para el desarrollo de la plántula.

\section{AGRADECIMIENTOS}

Queremos expresar nuestro reconocimiento al Dr. Hipólito Medrano, por facilitarnos el equipo del Laboratorio de Fisiología Vegetal de nuestra Facultad, y al Dr. Fabio Garbari por su amabilidad al suministrarnos información sobre $\mathrm{Br}$. fastigiata de Córcega y Cerdeña.

\section{BIBLIOGRAFIA}

CADEVALL, J. -1913-1937- Flora de Catalunya. Barcelona.

CAMEFORT, H. -1977- Morphologie des végétaux vasculaires. Cytologie, anatomie, adaptations. Paris.

CHOUARD, P. -1931- Révision de quelques genres et sous-genres de Liliacées bulbeuses, d'après le développement dè l'appareil végétatif (Scilla, Endymion, Hyacinthus). Bull. Mus. Nat. Hist. Natur. 2a s. III (1) : 176-180.

CHOUARD, P. -1933- Types de développement de l'appareil végétatif chez les Scillées. Ann. Sc. Natur. Bot. ser. 10,13:131-306.

DAMBOLDT, J.; MELZHEIMER, V. -1976- Brimeura fastigiata (Viv.) Chouard (Liliaceae) neu für die flora Griechlands. Bot. Jahrb. Syst. 95 (3) : 334-338.

GARBARI, F. -1966- Alcune osservazioni sul genere Brimeura Salisb. (Liliaceae). Nuovo Gior. Bot. Ital. 73 (1-6):324-325.

GARBARI, F. -1970- Il genere Brimeura Salisb. (Liliaceae). Atti. Soc. Tosc. Sci. Nat. Mem. Serie B $77: 12-36$.

GAUSSEN, H.; LEROY, J.F.; OZENDA, P. -1982- Précis de Botanique. 2. Végétaux Supèrieurs. Paris.

HEYWOOD, V.H. -1980- Brimeura. In T.G. TUTIN \& al. (eds.). Flora Europaea 5. Cambridge.

LLORENS, LL. -1984- Notas florísticas Baleáricas. Fol. Bol. Misc. 4:55-58.

MARTINOLI, G. -1949- Differenti livelli di vegetazione ai quali appartiene Hyacinthus fastigiatus Bert. Nuovo Gior. Bot. Ital. 56:679-689.

MOLERO, J. -1975- Notas taxonómicas y fitogeográficas. Anal. Inst. Bot. Cavanilles 32 (2):349-361.

MONTSERRAT, P. -1953- Aportación a la flora de Menorca. Collec. Bot. 3 (3) : 399-418.

PIGNATTI, S. -1982- Flora d'Italia. Vol. 3. Bolonia

PORTA, P. -1887- Stirpium in insuli Balearicum anno 1885 collectarum enumeratio. Nuovo Gior. Bot. Ital. ser. 1, 19:276-325.

RODRIGUEZ, J.J. -1904- Florula de Menorca. pp. 129 Mahón. 
SPETA, F. -1982- Uber die abczenzunc und gliederung der gattung Muscari, und uber ihre bezichungen $z u$ anderen vertretern der Hyacinthaceae. Bot. Jahrb. Syst. 103 (2):247-291.

VOSA, C.G. -1979- Heterochromatic banding patterns in the chromosomes of Brimeura (Liliaceae). Pl. Syst. Evol. 132:141-148.

Dirección de los autores: Lab. de Botánica. Fac. de Ciències. Universitat de les Illes Balears. Mallorca. Baleares. 